\title{
Imagem Corporal e Autoestima da Adolescente Grávida: Uma revisão integrativa da literatura
}

\section{Body Image, Self-esteem of Pregnant Adolescents: An integrative literature review}

Márcio Tavares ${ }^{1} \bowtie$

D Ana Paula Sousa Santos ${ }^{1}$
[1] Escola Superior de Saúde da Universidade dos Açores, Ponta Delgada, Portugal

$\triangle$ marcio.fm.tavares@uac.pt

\section{Resumo}

Objetivo: A ocorrência de uma gravidez na adolescência pode trazer implicações que devem ser analisadas e avaliadas. O presente estudo tem como objetivo explorar a influência da gravidez na imagem corporal e autoestima das adolescentes.

Método: Síntese de resultados obtidos a partir de estudos identificados por recurso a metodologia baseada nos princípios de revisão integrativa para seleção e localização de estudos quantitativos e qualitativos. Estudos publicados e indexados a bases de dados de pesquisa eletrónica.

Resultados: Tendo em consideração os critérios de inclusão e exclusão definidos, foram identificados 3 estudos, cujos resultados foram agrupados em torno de três eixos: $i$ ) Perceção positiva, $i i)$ Dificuldade na autocrítica e iii) Perceção negativa. Os resultados demonstraram que a gravidez pode influenciar a imagem corporal e a autoestima da adolescente de forma positiva ou negativa, dependendo da forma como é feito o acompanhamento. Os riscos psicossociais da gravidez são inúmeros e conhecidos.

Conclusões: A problemática da gravidez na adolescência exige um estudo aprofundado, de forma a contribuir para o desenvolvimento de intervenções e a concertação de estratégias para a sua prevenção. A construção de um forte apoio social poderá ter como efeito uma imagem corporal positiva e um aumento da autoestima nas adolescentes grávidas, mas também poderá funcionar como um fator preventivo da gravidez pela redução de comportamentos de risco.

Palavras-chave: Gravidez, Adolescência, Imagem corporal; Autoestima

\begin{abstract}
Objective: The occurrence of pregnancy in adolescence may bring about implications that should be analyzed and assessed. This study aims to explore the influence of pregnancy on adolescent girls' body image and self-esteem.

Method: Synthesis of results obtained from studies identified using a methodology based on the principles of integrative review to select and locate quantitative and qualitative studies. Studies published and indexed in electronic search databases.

Results: Considering the defined inclusion and exclusion criteria, 3 studies were identified, whose results were grouped around three axes: $i$ ) Positive perception, ii) Difficulty in self-criticism, and iii) Negative perception. The results showed that pregnancy can influence the adolescent's body image and self-esteem positively or negatively, depending on how the monitoring is performed. The psychosocial risks of pregnancy are numerous and known.

Conclusions: The problem of teenage pregnancy requires a thorough study to contribute to the development of interventions and concerted strategies for its prevention. Building strong social support may have the effect of creating a positive body image and increased self-esteem in pregnant adolescents. It may also function as a preventive factor of pregnancy by reducing risk behaviors.
\end{abstract}

Keywords: Pregnancy, Adolescence, Body image, Self-esteem 


\section{INTRODUÇÃO}

O indicador mais poderoso daquilo que é uma família numa determinada época e sociedade é indissociável daquilo que é pensado como o ciclo vital e, mais amiúde, das questões da procriação, da gravidez, do nascimento e do desenvolvimento de um filho. Todavia, o significado e o valor que são atribuídos a estes fenómenos têm evoluído ao longo dos tempos e de acordo com os contextos socioculturais (Canavarro, 2001).

Se bem que a problemática da gravidez tem sido estudada por várias perspetivas e sob o óculo de várias ciências, pouco tem sido explorado sobre as questões e os significados que as mudanças corporais assumem na mulher e na forma como encaram a gravidez. É sabido que algumas destas mudanças podem ser difíceis de lidar ou muito desconfortáveis e que tendem a se acentuar com o evoluir da gestação. Para além disso, após o parto, o corpo não retoma as suas formas pré-gravídicas de imediato, podendo demorar algum tempo a ser como era, ou pode nunca mais voltar completamente a ser como era antes da gravidez. Embora estas mudanças sejam naturais, algumas mulheres podem ter dificuldade em manter uma imagem positiva do corpo durante e após a gravidez (Tavares, 2015). Na verdade, o ciclo gravídico-puerperal pode ser caracterizado por um período repleto de vulnerabilidades tanto emocionais como físicas, que se revestem por uma reformulação da autoimagem, relacionados com os desconfortos típicos de cada trimestre da gravidez e pelas alterações vividas no puerpério. Estas alterações, obrigam a profundas transformações do autoconceito da mulher, que podem originar sentimentos auto depreciativos, dado que as experiências emocionais referentes a esta fase, demostram-se como intensas e avassaladoras (Tavares, 2015).

Todavia, se associarmos a esta premissa o conceção do self, que não é mais do que a imagem que cada indivíduo tem de si, percebemos que o conceito individual é criado e constantemente atualizado a partir das interações que se estabelece com os outros, podendose afirmar que é uma construção social do autoconceito identitário, influenciado pelas expetativas e exemplos dos outros, razão que se diz que o self e o ambiente são socialmente construídos (Bluff, 2006).

Numa sociedade em que a imagem corporal tende a ser cada vez mais valorizada e em que os estereótipos de perfeição tendem a ser generalizados nos meios de comunicação social como influenciadores de novas tendências (Silva, et al., 2014; Martins \& Petroski, 2015), são ainda poucos os estudos que procuram a influência que a gravidez pode ter sobre a perceção da imagem corporal das adolescentes. De facto, se pensarmos que não há a 
possibilidade de se sair igual da experiência de uma gravidez e que nada voltará a ser como era antes após o seu término, importa compreender a perceção da imagem corporal entre adolescentes grávidas e o seu impacto na sua autoestima, razão pela qual optou-se por realizar a presente revisão integrativa da literatura.

\section{MÉTODO}

No sentido de sistematizar o estado do conhecimento sobre a imagem corporal das adolescentes grávidas, realizou-se uma revisão integrativa da literatura, baseada nos princípios metodológicos e nas orientações da Joanna Brigs Institute (2021) ${ }^{1}$, nomeadamente: $i$ ) formulação da questão para a elaboração da revisão integrativa da literatura; ii) especificação dos métodos de seleção dos estudos; iii) procedimento de extração dos dados; $i v$ ) análise e avaliação dos estudos incluídos na revisão integrativa da literatura; v) extração dos dados e apresentação da revisão/síntese do conhecimento produzido e publicado.

A presente revisão teve como questão norteadora "Qual a influência da gravidez na imagem corporal e autoestima das adolescentes?", que se constitui o centro do desenvolvimento de toda a revisão, nomeadamente, desde a organização da estratégia de pesquisa até à discussão dos resultados.

Tendo em mente a pergunta de partida, traçaram-se critérios de inclusão na investigação, que possibilitassem a localização e a seleção de estudos empíricos relevantes:

- População - Adolescentes Grávidas;

- Indicador - influência da gravidez na autoestima e na imagem corporal;

- Desenho - Estudos primários dentro do paradigma qualitativo ou quantitativo, entre os anos 1991 e 2021.

Ainda no decorrer deste trabalho, definiram-se como critérios de exclusão: estudos não indexados a bases eletrónicas de pesquisa ou estudos em outras línguas que não o português, o inglês, o francês ou o castelhano e estudos secundários.

\subsection{Estratégia de pesquisa}

A pesquisa para a seleção de estudos relevantes para este trabalho, foi efetuada em bases de dados eletrónicas ${ }^{2}$ (maio de 2021). Para tal, isolaram-se quatro conceitos:

\footnotetext{
${ }^{1}$ https://jbi.global/

${ }^{2}$ MedlineComplete; CinahlComplete; Cochrane Central Register of controlled trials; Cochrane data base of systematic reviews; Lybrary; 
1 - Imagem Corporal, 2 - Autoestima, 3 - Adolescentes, 4 - Gravidez

Após foram identificados um conjunto de sinónimos e de termos relacionados (cf. Tabela 1), de modo a obter-se uma expressão de pesquisa ${ }^{3}$.

\section{Tabela 1}

Conceitos da Expressão de Pesquisa

\begin{tabular}{c|c|c|c}
\hline Pesquisa por Resumo & Pesquisa por Resumo & Pesquisa por Título & Pesquisa por Título \\
\hline Conceito 1 & Conceito 2 & Conceito 3 & Conceito 4 \\
Imagem Corporal & Autoestima & Adolescent & Gravidez \\
Selfimage & Self-esteem & Adolescence & Gestação \\
Body image & Self concept & Puberty & Pregnancy \\
Corporal image & & Teenager & \\
\hline
\end{tabular}

Nota: termos pesquisados na língua portuguesa e língua inglesa

A revisão efetuada foi deliberadamente pensada para as questões da imagem corporal nas adolescentes grávidas. O processo de revisão e análise dos dados foi efetuado, em conjunto, por dois revisores, os autores.

\section{RESULTADOS}

Recorrendo a esta estratégia de pesquisa, identificaram-se apenas 5 referências a estudos nas diferentes bases de dados. Com base nos critérios de inclusão/exclusão estabelecidos para este estudo. Posteriormente, passou-se a selecionar os estudos identificados, num processo que incluiu três fases: $i)$ avaliação pelo título do estudo; ii) avaliação do resumo e, finalmente, iii) avaliação dos textos completos. No final deste processo foram incluídos somente 3 estudos (Figura 1).

\footnotetext{
Nursing \& Allied Health collection comprehensive; British Nursing Index; Fuente Académica; Wiley Interscience; Taylor and Francis; Elsevier; Lila; Scielo; Cochrane Methodology Register; InformatiomScience \& Technology Abstracts; MedicLatina.

${ }^{3}$ AB (Imagem Corporal or Selfimage or Body image or Corporal image) And AB (Autoestima or Self-esteem or Self-concept) And TI (Adolescent or Adolescence or Puberty or Teenager or Youngster) And TI (Gravidez or Gestação or Pregnancy) 
Figura 1

Processo de seleção dos estudos de investigação

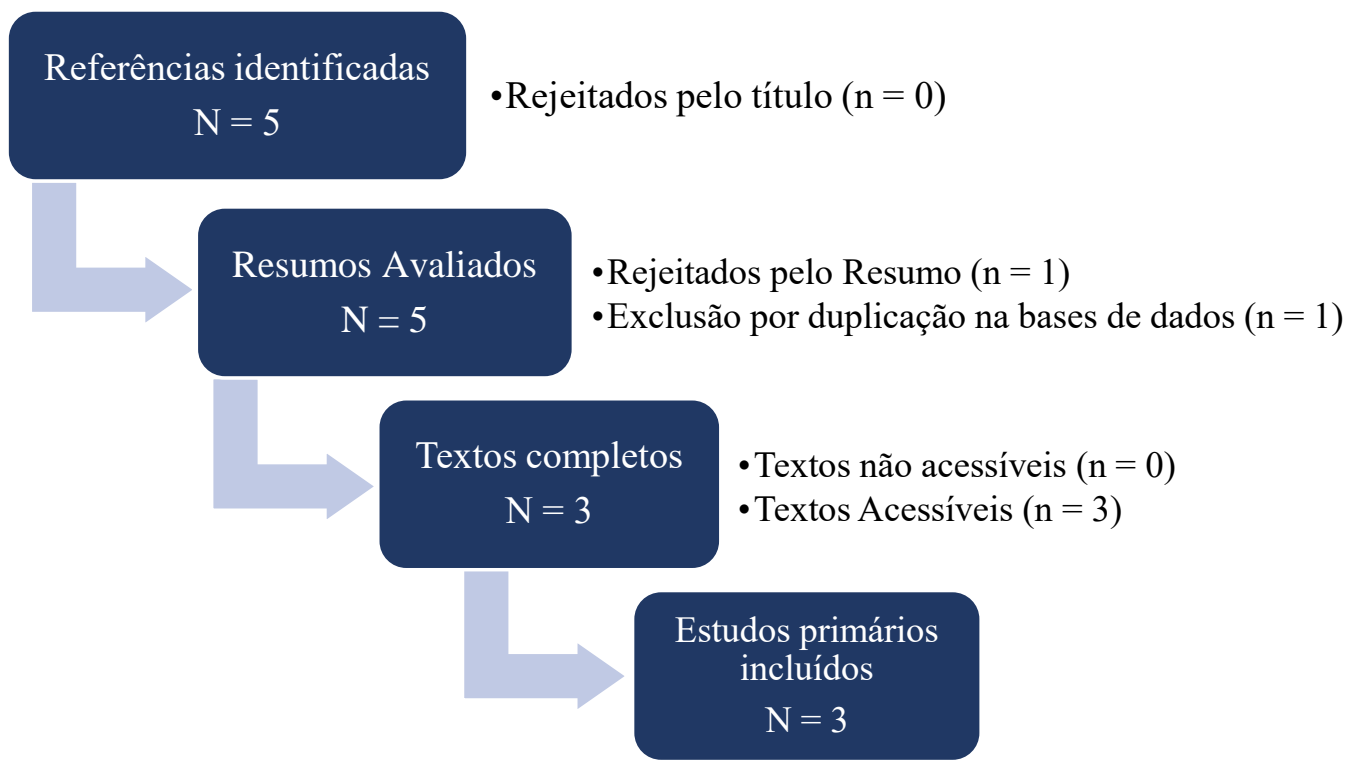

Procurou-se sistematizar, em tabelas, a informação recolhida dos estudos de investigação selecionados e publicados entre os anos de 1991 e 2016. Assim, na Tabela 2 encontram-se de forma resumida os estudos primários selecionados, os dados relativos aos seus autores, ano e país, participantes, orientações metodológicas e os resultados principais apontados nos estudos.

Para analisar os quadros de resumo da evidência recolhida, recorreu-se à análise de conteúdo, uma vez que nos permite realizar inferências, com base numa lógica explicitada, sobre as mensagens, cujas características foram inventariadas e sistematizadas, de acordo com a sua similaridade (Vala, 2009). Assim, analisando os quadros de resumo de evidência recolhida sobre o fenómeno em estudo, agregaram-se os resultados major em torno de três categorias (Tabela 3), tentaremos, a partir dos resultados dos três estudos analisados, explorar estas categorias de forma a obter as respostas pretendidas. 


\section{Tabela 2}

Resumo da evidência recolhida em estudos primários de investigação

\begin{tabular}{|c|c|c|c|c|}
\hline Autores/Ano/País & Participantes & Metodologia & Objetivos & Resultados Major \\
\hline $\begin{array}{l}\text { Matsuhashi e Felice } \\
\text { (1991) EUA }\end{array}$ & $\begin{array}{l}\mathrm{N}=86 \\
43 \text { adolescentes } \\
\text { grávidas do } 3^{\circ} \\
\text { trimestre } \\
43 \text { adolescente não } \\
\text { grávidas }\end{array}$ & Estudo quantitativo & $\begin{array}{l}\text { Comparar a perceção corporal de } \\
\text { adolescentes grávidas com } \\
\text { adolescentes não grávidas }\end{array}$ & $\begin{array}{l}\text { Quando comparadas com raparigas nunca grávidas, as } \\
\text { raparigas grávidas têm uma autoestima global mais } \\
\text { elevada, uma imagem corporal mais positiva, uma } \\
\text { autoidentidade mais segura, e sentem-se mais produtivas } \\
\text { como membro da família, mas parecem limitadas na } \\
\text { capacidade de autocrítica. }\end{array}$ \\
\hline $\begin{array}{l}\text { Stenberg e Blinn } \\
\text { (1993) EUA }\end{array}$ & $\begin{array}{l}14 \text { adolescentes } \\
\text { grávidas }\end{array}$ & $\begin{array}{l}\text { Qualitativa; análise } \\
\text { de diários pessoais de } \\
6 \text { semanas sobre as } \\
\text { questões: } \\
\text { "Os meus } \\
\text { pensamentos sobre } \\
\text { mim" } \\
\text { "Os meus } \\
\text { pensamentos sobre o } \\
\text { meu corpo" }\end{array}$ & $\begin{array}{l}\text { Explorar a estabilidade dos } \\
\text { sentimentos das adolescentes } \\
\text { sobre o self e a imagem corporal } \\
\text { durante a gravidez }\end{array}$ & $\begin{array}{l}\text { Após análise das entradas dos diários e atribuição de } \\
\text { pontos, numa escala de } 1 \text { a } 5 \text {, obtiveram-se os seguintes } \\
\text { resultados: } \\
\text { - Sentimentos sobre si própria - as palavras "nervosa", } \\
\text { "deprimida", "solitária" e "mal-humorada" apareceram } \\
\text { frequentemente; tiveram uma pontuação média global de } \\
2,18 \text {. } \\
\text { - Imagem corporal - as palavras } \\
\text { "gorda", "enorme" e "feia" foram as mais frequentes; } \\
\text { pontuação média } 2,40 \text {. } \\
\text { - Verificou-se um conflito entre o aumento de peso na } \\
\text { gravidez e a ênfase da cultura na magreza. } \\
\text { - As entradas do diário relacionadas com o self eram } \\
\text { mais variáveis do que as entradas da imagem corporal } \\
\text { (1,89 e } 1,56 \text { respetivamente). }\end{array}$ \\
\hline $\begin{array}{l}\text { Durmaz e Tufekci } \\
\text { (2016) Turquia }\end{array}$ & $\begin{array}{l}66 \text { adolescentes } \\
\text { grávidas com } \\
\text { idades entre os } 12 \mathrm{e} \\
\text { os } 19 \text { anos }\end{array}$ & $\begin{array}{l}\text { Pré-teste/pós-teste; } \\
\text { modelo quási- } \\
\text { experimental }\end{array}$ & $\begin{array}{l}\text { Determinar a influência da } \\
\text { formação em autocuidado dada } \\
\text { às adolescentes grávidas sobre a } \\
\text { capacidade de autocuidado, } \\
\text { autoestima e imagem corporal. }\end{array}$ & $\begin{array}{l}\text { As perceções da imagem corporal das adolescentes } \\
\text { grávidas apresentam diferenças estatísticas }(p<.001) \text {, os } \\
\text { seus níveis de autoestima }(p<.01) \text { aumentaram após } \\
\text { formação em autocuidado. }\end{array}$ \\
\hline
\end{tabular}


Tabela 3

Categorias e Subcategorias Evidentes nos Resultados Major no Total dos Três Estudos

\begin{tabular}{l|l|l}
\hline \multicolumn{1}{c|}{ Categorias } & \multicolumn{1}{|c}{ Subcategoria } & \multicolumn{1}{c}{ Estudos } \\
\hline \multirow{4}{*}{ Perceção Positiva } & Imagem corporal & $\begin{array}{l}\text { Matsuhashi e Felice (1991) } \\
\text { Durmaz e Tufekci (2016) }\end{array}$ \\
\cline { 2 - 3 } & Autoestima & \multirow{2}{*}{ Matsuhashi e Felice (1991) } \\
\cline { 2 - 3 } $\begin{array}{l}\text { Dificuldade na } \\
\text { Autocrítica }\end{array}$ & Autoidentidade mais segura & Stenberg e Blinn (1993) \\
\hline \multirow{2}{*}{\begin{tabular}{l} 
Perceção Negativa \\
\cline { 2 - 3 }
\end{tabular}} & Imagem corporal & \\
\cline { 2 - 3 } & Autoestima & \\
\hline
\end{tabular}

A primeira categoria que emergiu, denominada de Perceção Positiva, congrega a informação dos estudos que inclui uma visão positiva da imagem corporal, da autoestima e da autoidentidade. Na segunda categoria, denominada por Dificuldade na Autocritica, agrupou-se a evidência que demonstra que as adolescentes têm dificuldade em se autoapreciar e criticar. Por último, na categoria Perceção Negativa, procurou-se juntar os achados que reúnem uma visão negativa da imagem corporal e da autoestima.

\section{DISCUSSÃO}

A adolescência é uma fase da vida humana, caracterizada por inúmeras alterações psicológicas, sociais, físicas e metabólicas, que transformam o indivíduo (Medeiros, 2013). As alterações de comportamento, a necessidade de experimentar desejos e satisfazer curiosidades, de descobrir o corpo e o prazer sexual, podem favorecer atitudes e comportamentos inconsequentes, resultando em situações como a gravidez não planeada. Tais situações, podem conduzir a sentimentos de perda de identidade, da expectativa do futuro, da confiabilidade e da proteção da família (Batista et al, 2021).

É nesta fase que se estabelecem referências para uma parte da sua vida adulta, definidas na família, na escola e na sociedade, onde os adolescentes se inspiram para o desenvolvimento da sua personalidade. Se se pensar que a gravidez na adolescência pode ser considerada um indicador de desenvolvimento de uma sociedade, então percebe-se, rapidamente, que estamos perante um problema complexo e multifatorial, com relação íntima com a pobreza, a baixa escolaridade, com riscos importantes para a saúde materna e infantil, desde logo, aquela que é mais grave - a mortalidade materna, neonatal e infantil (Batista et al., 2021). Em Portugal este continua a ser um problema de saúde pública, pois 
de acordo com o Instituto Nacional de Estatística em $2020^{4}$, foram 1763 as crianças que nasceram de mães adolescentes.

As mudanças que a adolescente assiste no seu corpo em virtude do seu normal desenvolvimento, exigem ajustamento e aceitação. As que vêm associadas à gravidez, são mais complexas e surgem em um curto espaço de tempo, podendo repercutir negativamente na saúde física e mental das adolescentes, com influência direta na perceção positiva que o próprio tem de si, da sua imagem corporal, da sua autoestima e da sua identidade (Matsuhashi \& Felice, 1991; Durmaz \& Tufekci, 2016). Significa então, que a Gravidez na adolescência poderá conduzir ao desenvolvimento de problemáticas que transcendem as típicas nesta fase de desenvolvimento.

Contudo, o desenvolvimento de programas formativos direcionados para o autocuidado, parece ter uma influência positiva sobre as adolescentes. O estudo quasiexperimental desenvolvido por Durmaz e Tufekci (2016), testou a eficácia de um programa de formação direcionado a adolescentes grávidas. Recorrendo a pré e pós-testes, verificaram que as 66 adolescentes grávidas, revelaram aumento dos níveis de satisfação quer com a imagem corporal, quer com a autoestima, após a frequência de uma em autocuidado. Neste sentido, os autores sugerem a concertação de projetos/políticas/programas em que o autocuidado, a autoestima e a imagem corporal seja melhorada e aumentada através de práticas de cuidados centrados na família, desde o período de pré-gravídico até ao período pós-parto.

Transpondo para a realidade portuguesa, seria de considerar a inclusão destas temáticas não Programa Nacional para a Vigilância da Gravidez da Direção Geral de Saúde de forma a garantir às adolescentes grávidas, um acompanhamento sistemático, no sentido de as ajudar a lidar com questões essenciais relacionadas com a gravidez, mas focando nas questões próprias que lhe dizem respeito e que lhes preocupa. Por outro lado, a inclusão destas temáticas nestes programas seria uma forma de sensibilizar os profissionais para as mudanças de imagem, do self e do corpo da adolescente grávida, para que possam intervir de modo a favorecer a aceitação do novo eu e da nova condição.

No estudo conduzido por Babington et al. (2015), foi possível perceber que menores níveis de autoestima e apoio social reduzido, eram mais frequentes entre adolescentes que engravidaram. No polo oposto, verificaram que as adolescentes com maior autoestima e apoio social, têm menor probabilidade de engravidar. Esta é sem dúvida uma descoberta

\footnotetext{
${ }^{4}$ https://www.ine.pt/xportal/xmain?xpid=INE\&xpgid=ine indicadores\&indOcorrCod=0001541\&contexto=pgi\&selTab=tab10\&xlang=pt 
importante pois demonstra a necessidade de se investir em medidas promotoras da autoestima e de promover maior apoio social às adolescentes, como forma de prevenir gravidezes. Neste sentido, a educação sexual nas escolas, os gabinetes de apoio ao adolescente, as famílias e as comunidades podem e devem desempenhar um papel relevante neste sentido. O estabelecimento de um plano de ação de literacia para a saúde, por exemplo, pode demonstrar-se eficaz no apoio às adolescentes, munindo-as de estratégias de "empowerment" e estratégias de coping que as auxiliem nas suas decisões.

Uma baixa autoestima pode acarretar riscos, nomeadamente no funcionamento social do indivíduo (Pedro \& Peixoto, 2006; Quiles \& Espada, 2009), conduzindo a comportamentos de risco, que podem se caracterizar pela manutenção de relações sexuais desprotegidas, maior número de parceiros sexuais e maior risco de gravidez (Boden \& Horwood, 2006). No entanto, parece que os adultos enquanto adolescentes, mantinham uma autoestima baixa, não registam comportamentos sexuais de risco, o que parece ser explicado pelo contexto psicossocial dentro do qual a autoestima destas mulheres se desenvolve. Nesta medida, o apoio familiar aquando de uma gravidez na adolescência parece favorecer o desenvolvimento da autoestima (Rodrigues et al, 2010).

Todavia, num estudo recente desenvolvido por Wendt e Appel-Silva (2020), parece que as práticas parentais não demonstram associações robustas com a autoestima. Ainda assim, não pode ser descurada que as práticas parentais adequadas terão uma influência positiva sobre a personalidade do indivíduo, com desfechos relevantes no desenvolvimento socioemocional de crianças e adolescentes, levando a que haja menor sintomatologia depressiva e maior autoestima (Garber et al., 1997).

A imagem corporal considerada um conceito multidimensional (Cash \& Smolak, 2012), pode ser entendida como a figura mental que o próprio se vê com o tamanho e forma do corpo (e.g., esquemas da aparência) (Mendes et al., 2019), a ideia de que a gravidez pode influenciar negativamente as adolescentes, não parece descabida. Sendo a adolescência uma fase de adaptação às alterações que ocorrem de forma abrupta (Stenberg \& Blinn, 1993, Medeiros, 2013), tê-las associadas às alterações que surgem devido à gravidez, pode ser confuso e avassalador.

$\mathrm{Na}$ verdade, parece que as pessoas cujos esquemas sobre a aparência traduzem níveis superiores de investimento, tendem a focar-se atentamente na aparência e envolvem-se em comportamentos que permitam mantê-la ou melhorá-la. Verifica-se igualmente que revelam maiores níveis de ansiedade social e de perturbação alimentar e mais suscetíveis ao sofrimento (Cash et al, 2004). Quando acompanhada de evidência, há que refletir sobre este 
significado e procurar compreender que medidas deverão ser implementadas para controlar a perceção negativa da imagem corporal e da autoestima (Stenberg \& Blinn, 1993).

Stenberg e Blinn (1993), desenvolveram um estudo que analisou diários escritos de 14 adolescentes grávidas, com idades entre os 15 e os 19 anos e uma média de idades de 16 anos. Foi solicitado a estas adolescentes, que escrevessem diariamente durante seis semanas sobre duas questões: "Os meus pensamentos sobre mim" e "Os meus pensamentos sobre o mеи согро". Após as seis semanas, foram analisadas as entradas e a qualidade afetiva das entradas, classificada numa escala de 1 (muito negativa) a 5 (muito positiva), de acordo com critérios previamente definidos. Verificou-se que a qualidade média afetiva das entradas relacionadas com a imagem corporal foi de 2,40 pontos, tendo sido utilizados termos como "gorda", “enorme" e "feia". Na mesma escala, verificou-se que os sentimentos sobre si própria tiveram uma pontuação média global de 2,18, sendo que as palavras mais frequentes foram "nervosa", "deprimida", "solitária" e "mal-humorada".

A verdade é que o culto pela imagem corporal ideal, cultivada nos media, não facilita o processo adaptativo à imagem corporal em constante alteração (Rumsey \& Harcourt, 2012). A cultura da magreza, conduz ao eterno conflito de aumento de peso na gravidez, que não é entendido como um acontecimento normal, mas antes como um “engordar". Salientese que ainda no estudo apresentado por estes autores, as entradas do diário relacionadas com o self eram mais variáveis (grande média de alterações, 1,89) do que as entradas da imagem corporal $(1,56)$.

Matsuhashi e Felice (1991) conduziram um estudo com o intuito de perceber se haveria diferença na perceção corporal de adolescentes grávidas com adolescentes nunca grávidas. Para isso, recorreram a dois grupos: um das adolescentes grávidas $(n=43)$ e outro das adolescentes nunca grávidas $(n=43)$ que correspondessem em termos de idade, raça, estatuto socioeconómico e desenvolvimento pubertário. Foi possível verificar que as adolescentes grávidas apresentavam uma autoestima global mais elevada, uma imagem corporal mais positiva, uma identidade mais segura e sentiam-se mais produtivas como membros integrantes da sua família. Todavia, também essas adolescentes pareciam limitadas na capacidade de autocrítica. De acordo com o estudo de Babington et al. (2015) as adolescentes grávidas que planeiam a sua gravidez, registam índices de autoestima superiores àquelas que engravidam de forma acidental. Se se pensar que na base da decisão de engravidar poderá estar a ideia de que a gravidez foi planeada com a sua "alma gémea", então percebemos, que a problemática da gravidez na adolescência associada à autoestima exige um estudo ainda mais aprofundado (Gomes \& Speizer, 2010), como seja conhecer os 
fatores externos que influenciam na decisão das adolescentes em engravidar, reconhecendo a pressão dos pares e o seu poder persuasivo nas decisões.

Estas descobertas são importantes, pois poderão contribuir para o desenvolvimento de intervenções e a concertação de estratégias para prevenir a gravidez na adolescência e a promoção da autoestima. O aumento da autoestima e a construção de um forte apoio social poderão ter como efeito a prevenção da gravidez e a redução de comportamentos de risco na adolescência, no entanto, mesmo que tal não se revele útil, poderá enformar-se como intervenções eficazes destinadas ao empoderamento. Durmaz e Tufekci (2016) defendem que no caso de a adolescente ainda assim, decidir por engravidar, tais estratégias permitirão o desenvolvimento da sua autoestima, balizada num ambiente de apoio. Estes autores argumentam ainda que a capacitação para encetar esta aventura, com conhecimento, valorizando a sua imagem corporal e convergindo para o desenvolvimento da sua autoestima, ao invés de concorrer para a sua desvalorização e auto-depreciação.

\section{CONCLUSÕES}

A autoimagem, autoestima, gravidez e adolescência são conceitos que, relacionados entre si, são constitutivos de temáticas que exigem exploração, conhecimento importante para o desenvolvimento de estratégias que permitam a prevenção de comportamentos de risco. Assim, a importância deste tema justifica-se pela necessidade de se perceber qual o impacto que uma gravidez pode ter na autoimagem e autoestima das adolescentes, dado que influencia todo o seu futuro.

Desta revisão integrativa de literatura, resultaram três categorias: Perceção Positiva, Dificuldade na Autocrítica e Perceção Negativa. De facto, parece que uma imagem corporal negativa e uma baixa autoestima influencia de forma negativa os comportamentos das adolescentes, explicados pelo contexto psicossocial dentro do qual se desenvolve. Por outro lado, percebe-se que as adolescentes que têm apoio familiar, tendem a apresentar melhores níveis de imagem corporal e autoestima, bem como aquelas que usufruem de formação ou acompanhamento.

A continuação dos estudos e uma gravidez planeada, parece favorecer níveis superiores de autoestima. É evidente a necessidade de investir em investigação nesta área, dado à escassez de estudos que explorem estas preocupações. A aplicação de escalas, questionários e a condução de entrevistas, são essenciais para conhecer aprofundadamente este fenómeno. 
Não obstante a investigação sobre a gravidez na adolescência associada à imagem corporal e autoestima seja relevante, os seus resultados ainda não conduzirem a recomendações consistentes para a promoção da imagem corporal e autoestima, exigindo-se um estudo ainda mais aprofundado, como seja conhecer os fatores externos que influenciam na decisão das adolescentes em engravidar, reconhecendo a pressão dos pares e o seu poder persuasivo nas decisões. Também, estudar a perspetiva do pai adolescente e a sua autoestima, considerando os estereótipos vigentes na sociedade, parece ser territórios que deverão ser, igualmente, mais explorados e que se revelam como pontos de interesse para investigações futuras.

Como limitação desta revisão, pode ser apontado o facto de o processo de revisão não ter sido efetuado de forma independente, embora o número reduzido de trabalhos não pareça ter deixado dúvidas para a inclusão dos estudos. Ainda assim, espera-se ter contribuído para estimular outros investigadores a investirem nesta área de interesse, uma vez que a imagem corporal e a autoestima são componentes fundamentais da saúde humana e, portanto, promovê-las é um dever dos profissionais envolvidos no cuidado humano.

Conflitos de interesse: Os autores declaram a inexistência de conflitos de interesse na realização do trabalho. $\mathrm{O}$ autor de correspondência pertence à equipa Editorial, no entanto, não desempenhou qualquer papel de intervenção no processo de revisão por pares double-blinded.

Fontes de financiamento: Não existem fontes externas de financiamento para a realização deste artigo.

Agradecimentos: Os autores não têm quaisquer apoios a declarar.

\section{Referências}

Babington, L.M.; Malone, L.; Kelley, B.R. (2015). Perceived social support, self-esteem, and pregnancy status among Dominican adolescents. Applied Nursing Research, 28, 121-126. Doi: http://dx.doi.org/10.1016/j.apnr.2014.08.001

Batista, M.H.J.; Lino, D.B.; Sousa da Silva, M.;Costa M.D.C; Rocha,M.A.; Nunes, R.S. (2021). Gravidez na adolescência e a assistência de enfermagem: uma abordagem sobre os riscos à saúde maternal e neonatal. Saúde coletiva, 61(11), 4978-4983. https://doi.org/10.36489/saudecoletiva.2021v11i61p4978-4989

Bluff, R. (2006). Grounded Theory. In Cluett, E.R., \& Bluff, R.. Principles and practice of research in midwifery (2nd ed.). Philadelphia: Churchill Linvingstone Elsevier.

Boden, J.M.; Horwood, L.J.; (2006). Self-Esteem, Risky Sexual Behavior, and Pregnancy in a New Zealand Birth Cohort. Archives of Sexual Behavior, 35, 549-560. https://doi.org/10.1007/s10508-006-9060-4 
Canavarro, M. C. (2001). Psicologia da gravidez e da maternidade. Coimbra: Quarteto Editora.

Cash, T. F., \& Smolak, L. (2012). Body Image: A handbook of science, practice, and prevention (2nd ed.). Guilford Press.

Cash, T. F., Melnyk, S. E., \& Hrabosky, J. I. (2004). The assessment of body image investment: An extensive revision of the Appearance Schemas Inventory. International Journal of Eating Disorders, 35(3), 305-316. https://www.semanticscholar.org/paper/The-assessment-ofbody-image-investment\%3A-an-of-the-CashMelnyk/84be80b139128d21f991b6a9c3234d315a700760

Durmaz, G.B.; Tufekci, F.G. (2016). The Self-Care Training is Increasing Body Image Perception Self-Esteem and Self-Care Abilities in Pregnant Adolescents: A Pretest-Posttest QuasiExperimental Study. International Journal of caring Sciences, 9(3). http://www.internationaljournalofcaringsciences.org/docs/16_1-tufekci_originial_9_3.pdf

Garber, J., Robinson, N. S., \& Valentiner, D. (1997). The relation between parenting and adolescent depression: Self-worth as a mediator. Journal of Adolescent Research, 12(1), 1233.https://doi.org/10.1177/0743554897121003

Gomes, K.R.O.; Speizer, I.S. (2010). Longitudinal study on self-esteem among recently pregnant Brazilian adolescents. Journal of Reproductive and Infant Psychology, 28(4), 359-371. https://doi.org/10.1080/02646831003587361

Martins, CR; Petroski, EL (2015). Insatisfação com a imagem corporal em adolescentes do sexo feminino de uma cidade de pequeno porte: prevalência e correlações. Motricidade, 11(2), 12. https://doi.org/10.6063/motricidade.3670

Matsuhashi, Y.; Felice, M.E. (1991). Adolescent body image during pregnancy. The Journal of adolescent health, 12 (4), 313-315. https://doi.org/10.1016/0197-0070(91)90005-7

Medeiros, T. (2013). Adolescência: Desafios e Riscos. Letras Lavadas

Mendes, J., Rego, R., \& Pereira, V. (2019). Tradução e adaptação da escala CARSAL/CARVAL para Portugal: Estudo psicométrico. PSICOLOGIA, 33(1), 47-54. https://doi.org/10.17575/rpsicol.v33i1.1425

Moyano,. N.; Granados, R.; Dúran, C.A., Galarza, C. (2021). Self-Esteem, Attitudes toward Love, and Sexual Assertiveness among Pregnant Adolescents. International Journal of Environmental Research And Public Health, 18(3) 1270. https://doi.org/10.3390/ijerph18031270

Pedro, P. e Peixoto, F. (2006). Satisfação profissional e autoestima em professores dos $2^{\circ}$ e $3^{\circ}$ ciclos do Ensino básico. Análise Psicológica, 2 (25), 247-262. https://doi.org/10.14417/ap.167

Quiles, M.J.; Espada, J.P. (2009). Educar para a uto-estima: Propostas para escolas e par o tempo livre. (2sd ed.). K editora.

Rodrigues, J.E.; Nascimento, M.V.; Filho, A.V.D.; Pontes-Barros, J.F; Nunes de Magalhães, M.; Gomes, A.F.P.; Santos, A.C.R.S. (2010). Avaliação da autoestima de adolescentes gestantes de baixa renda assistidas pelo Núcleo de Apoio à Saúde da Família. Conscientiae saúde, 9(3), 395-401. https://www.redalyc.org/pdf/929/92915180008.pdf

Rumsey, N. \& Harcourt, D. (2012). The Oxford handbook of the psychology of appearance. Oxford Library of Psychology

Silva, M.L.; Taquette, S.R.; Coutinho, E.S. (2014). Sentidos da Imagem Corporal de Adolescentes no ensino fundamental. Revista Saúde Pública, 48(3).https://doi.org/10.1590/S00348910.2014048005083

Stenberg, L.; Blinn, L. (1993). Feelings about Self and Body during Adolescent Pregnancy. Families in Society: The Journal of Contemporary Social Services, 74 (5), 282-290. https://doi.org/10.1177/104438949307400503

Tavares, M.F.M. (2015). Transição para a parentalidade e a saúde mental no puerpério: significados para a mulher em risco de Depressão Pós-Parto. (Tese de doutoramento, ICBAS, Universidade do Porto, Porto, Portugal). https://repositorioaberto.up.pt/bitstream/10216/78946/2/35026.pdf 
Vala, J. (2009). A análise de conteúdo. In, A. Silva, \& J. M. Pinto (Eds.).Metodologia das ciências sociais. 15th ed. (101-128). Afrotamento.

Wendt, G.W.; Appel-Silva, M. (2020). Práticas Parentais e Associações com Autoestima e Depressão em Adolescentes. Pensando famílias, 24(1), 224-238. http://pepsic.bvsalud.org/pdf/penf/v24n1/v24n1a16.pdf 